\title{
Timing of Entry under Externalities
}

\author{
Ping Lin and Kamal Saggi*
}

May 7, 2001

\begin{abstract}
A bstract
This paper constructs a model where two firms simultaneously choose their time of entry into a market. Under sequential entry, the second entrant is assumed to face a lower entry cost because of positive externalities from the first firm's entry. The model generates sequential entry if the magnitude of the externality is large relative to the post-entry duopoly profit, and simultaneous entry otherwise. In a sequential entry equilibrium, the first entrant fares better than the second and the second entrant does not necessarily enter too late from the viewpoint of social welfare. When firms have different costs of production, the efficient firm is more likely to enter first.

Keywords: Entry, Externality, Duopoly

J EL Classification: L13, D43, C72
\end{abstract}

\section{Introduction}

Initial entrants into a market often generate positive externalities for subsequent entrants: for example, the latter can lower their cost of entry by avoiding mistakes made by the former. The presence of such cost-lowering externalities for late comers makes it attractive for firms to postpone their entry. In such a situation,

${ }^{*}$ Lin (corresponding author): Department of Economics, Lingnan University, Hong Kong, plin@ln.edu.hk. Saggi: Department of Economics, Southern Methodist University, Dallas, TX 75275-0496, ksaggi@mail.smu.edu. We thank Ken Hendricks, Hideo Konishi, Nikos Vettas, Shlomo Weber, and two anonymous referees for valuable comments and suggestions. All errors are our own. We also thank Lingnan University for providing financial support. 
each potential entrant faces a trade-off between entering earlier than its rivals and enjoying monopoly status till additional entry occurs versus waiting and entering later in order to lower its entry cost. This paper formalizes this trade-off to endogenize the pattern of entry in a strategic setting.

One scenario where cost lowering externalities are likely to be significant is when firms make decisions regarding entry into foreign markets. For example, China recently underwent significant economic liberalization and foreign firms are not particularly well informed about the Chinese market. The experience of initial entrants into China can benefit subsequent entrants by helping them avoid costly mistakes. ${ }^{1}$ By transferring technology, training workers, and setting up research centers in China, initial investors may also lower the cost of establishing subsidiaries for future investors by making it easier for them to locate qualified personnel, local suppliers, and partners for joint ventures. ${ }^{2}$

Cost-lowering externalities may also arise when firms introduce a product about which consumers lack information: promotional efforts (advertising and introductory sales etc.) by the first entrant serve the important function of informing consumers about the nature of the product and benefit future entrants by lowering their corresponding expenditures. For example, when it first began service, Federal Express invested heavily in conveying to consumers the meaning of absolutely-positively overnight delivery whereas other overnight delivery firms that entered after FedEx did not have to make similar investments.

To capture the above ideas and study the effect of a "wait-and-see" strategy on equilibrium entry patterns, we construct a model in which two firms simultaneously choose their timing of entry into a market. In accordance with the motivation of the paper, we assume that the first firm's entry results in positive externalities that lower the second entrant's cost of entry. Furthermore, the cost

\footnotetext{
${ }^{1}$ Aitken, Hanson, and Harrison (1997) find empirical evidence that multinational firms who penetrate foreign markets reduce entry costs for other potential entrants.

${ }^{2}$ For example, while negotiating its entry into the Chinese market, Chubb Corporation, agreed to set up an 'insurance university' in return for market access. In the automobile industry, General Motors Corporation has been involved in similar efforts (Business W eek, 1996).
} 
reduction enjoyed by the second entrant increases continuously with the firm's waiting time. That is, the longer the second entrant delays its entry, the more it learns about the new market environment from studying the first entrant's experience, and the lower its entry cost. However, while the second entrant pays a lower entry cost, delayed entry also means forgone profits. This trade-off between a lower entry cost and foregone profits can generate simultaneous or sequential entry equilibria for different parameter values. When foregone profits are big relative to the cost lowering externality, simultaneous entry is the unique equilibrium. When the converse is true, we obtain a sequential entry equilibrium where the first firm jumps into the market instantaneously whereas the second firm enters after an optimal waiting period. Although the second entrant pays a lower entry cost, it receives a lower lifetime payoff in equilibrium than the first entrant. The sequential entry equilibrium also exhibits some interesting comparative statics which stem from one common mechanism: factors that delay the second firm's entry confer an indirect benefit upon the initial entrant by extending its monopoly status. Consequently, stronger positive externalities or a higher entry cost may increase the first entrant's payoff.

When firms are asymmetric in terms of their marginal costs of production, an important question is whether the low-cost firm necessarily enters first. Intuition suggests that since it faces a higher opportunity cost of waiting, the low-cost firm has a greater incentive to enter first. However, given that the high-cost firm enters first, it may be in the interest of the low-cost firm to delay entry because of the positive externality from initial entry. Our model shows that whenever the highcost firm entering first is a Nash equilibrium, the low-cost firm entering first is also a Nash equilibrium. In addition, we isolate a range of parameter values for which the low-cost firm entering first is the only equilibrium. Therefore, we claim that the sequential entry equilibrium in which the low-cost firm entering first is more likely. ${ }^{3}$ It is also shown that total industry profits are higher when the low-cost

\footnotetext{
${ }^{3} \mathrm{~A}$ casual perusal of the empirical evidence regarding foreign investment suggests that bigger firms usually do enter foreign markets first.
} 
firm enters first.

We also investigate socially desirable entry times and show that, relative to the social optimum, the second firm does not necessarily enter too late. Whether or not its entry time exceeds the socially optimal entry time depends upon whether the additional social surplus (social incentive for entry) generated by its entry exceeds the second entrant's share of duopoly profits (private incentive for entry).

Endogenous pattern of entry has received some attention in recent literature, but most existing work focuses on the demand side. Appelbaum and Weber (1994) develop a model in which two firms simultaneously choose between entering a market early versus later in the face of demand uncertainty. ${ }^{4}$ Maggi (1996) analyzes strategic investment in a new market also under demand uncertainty, where firms face a trade-off between commitment and flexibility. Our paper differs from these work in three main respects. First, whereas in these models, the advantage of being late stems from the resolution of uncertainty at the end of the first period (therefore this advantage is independent of the players' strategies), in our model a firm benefits from delaying entry only if its rival enters before it. Second, we determine entry times endogenously as opposed to the two period framework utilized in the literature. This feature of our model permits an investigation of the properties of the optimal waiting period. Finally, we also consider entry decisions of asymmetric firms, something that has not received much attention in the literature.

The paper is organized as follows. In section 2 we set up the basic model. In section 3, we derive the equilibrium entry patterns and study their properties. Next, in section 4, we consider the case of asymmetric firms. Section 5 examines whether private entry times differ from socially optimal ones. Section 6 concludes.

\footnotetext{
${ }^{4}$ See Rob (1990 and 1992) for analyses of informational externalities that reveal the state of demand to potential entrants in a dynamic competitive model.
} 


\section{The Basic M odel}

There are two identical firms who simultaneously choose their time of entry into a market at $t=0$. We assume that firms are able to commit to their entry times. Denote firm $i$ 's entry time by $t_{i}, 0 \leq t_{i}<\infty, i=1,2$. Firm $i$ 's entry cost is given by

$$
F_{i}=\left\{\begin{array}{ll}
F & \text { if } t_{i} \leq t_{j} \\
F e^{-\gamma\left(t_{\mathrm{i}}-t_{\mathrm{j}}\right)} & \text { if } t_{i}>t_{j}
\end{array}\right\}
$$

That is, if firm $i$ enters the market before firm $j$, then it pays a fixed cost $F$. If, however, it enters the market after firm $j$ then it pays a lower entry cost $F e^{-\gamma\left(t_{i}-t_{j}\right)}$, where $\gamma>0$ is the externality parameter. Our formulation above captures the notion that the second firm to enter pays a lower cost of entry because of externalities generated by the first firm's entry and the longer it waits, the lower its cost of entry. Note that the cost of entry depends upon the relative entry times of the two firms and does not exogenously decline over time and that the marginal value of waiting declines with time. This formulation is to be contrasted with the technology adoption literature where adoption cost declines exogenously over time (see Reinganum, 1981a, 1981b).

If a firm enters first, it earns flow monopoly profits (denoted by $m$ ) till the other firm enters. When both firms are in the market, each earns flow duopoly profits denoted by $\pi$. For a given pair $\left(t_{1}, t_{2}\right)$, let $V_{i}\left(t_{1}, t_{2}\right)$ denote firm $i$ 's sum of discounted profits, and $\rho$ the discount factor. We have

$$
V_{i}\left(t_{1}, t_{2}\right)=e^{-\rho t_{\mathrm{i}}}\left[\frac{m}{\rho}-F\right]+\left[\frac{\pi-m}{\rho}\right] e^{-\rho t_{\mathrm{j}}} \quad \text { if } t_{i}<t_{j}
$$

and

$$
V_{i}\left(t_{1}, t_{2}\right)=e^{-\rho t_{i}}\left[\frac{\pi}{\rho}-F e^{-\gamma\left(t_{i}-t_{j}\right)}\right] \quad \text { if } t_{i} \geq t_{j} .
$$

Assumption $1 \pi>\rho F$. 
The above assumption implies that simultaneous entry is profitable for both firms and it must hold for simultaneous entry to be even a possibility.

\section{Equilibrium Entry Patterns}

First note that if $t_{1} \geq t_{2}$,

$$
\frac{\partial V_{1}\left(t_{1}, t_{2}\right)}{\partial t_{1}}=\left[-\pi+(\rho+\gamma) F e^{-\gamma\left(t_{1}-t_{2}\right)}\right] e^{-\rho t_{1}}
$$

So, if $\pi \geq(\rho+\gamma) F, V_{1}\left(t_{1}, t_{2}\right)$ decreases with $t_{1}$ when $t_{1} \geq t_{2}$. In this case, firm 1 does not benefit from waiting; the magnitude of the positive externality $(\gamma)$ is too small relative to the duopoly profit $\pi$. Consequently, firm 1 chooses to enter no later than firm 2.

Further, note that if $t_{1}<t_{2}$,

$$
\frac{\partial V_{1}\left(t_{1}, t_{2}\right)}{\partial t_{1}}=[-m+\rho F] e^{-\rho t_{1}}<0
$$

Under Assumption 1, $V_{1}\left(t_{1}, t_{2}\right)$ is strictly decreasing in $t_{1}$ for $t_{1} \leq t_{2}$. That is, if firm 1 enters no later than firm 2 , it enters at $t=0$. Since a firm's entry cost declines only if its rival enters before it, the first entrant has no incentive to delay its entry.

Therefore, if $\pi \geq(\rho+\gamma) F, t_{i}=0$ is a (strictly) dominant strategy for both firms and simultaneous entry at time 0 is the unique Nash equilibrium. On the other hand, if $(0,0)$ is a Nash equilibrium, it must be that $\frac{\partial V_{1}(0,0)}{\partial t_{1}} \leq 0$, which is equivalent to $\pi \geq(\rho+\gamma) F$ by equation (3.1). The following result then obtains.

Proposition 1: Simultaneous entry by both firms at $t=0$ is the unique pure strategy Nash equilibrium if and only if $\pi \geq(\rho+\gamma) F$.

The intuition for this result is clear. Given that its rival enters at $t=0$, each firm faces the decision of either entering at $t=0$ or delaying entry to lower its entry cost. The marginal benefit of delaying entry by a small time $d t$ is given by $(\rho+\gamma) F-$ the reduction in cost of entry. The marginal cost of postponing 
entry equals instantaneous duopoly profits $\pi$. When $\pi \geq(\rho+\gamma) F$ waiting is not worthwhile for the firms since the loss in profits incurred by delaying entry dominates the cost savings enjoyed due to decrease in entry cost.

It is clear then that the sequential entry equilibrium can be obtained only when duopoly profits post entry are small relative to the magnitude of the externality and the cost of entry. Therefore, next we consider the case where $\pi<(\rho+\gamma) F$.

Suppose that the first entrant enters at time $t_{1}$. By (3.1) and symmetry of firms, if $t_{2}>t_{1}$, we have

$$
\frac{\partial V_{2}\left(t_{1}, t_{2}\right)}{\partial t_{2}}=0 \Rightarrow \pi=(\rho+\gamma) F e^{-\gamma\left(t_{2}-t_{1}\right)}
$$

Note that the marginal benefit of waiting declines over time whereas the marginal cost of waiting is constant. Therefore, when the marginal benefit of waiting equals the marginal cost, the second firm enters the market. Equation (3.3) can be solved for the optimal waiting period $t^{*}$ :

$$
t^{*}=\frac{1}{\gamma} \ln \left[\frac{F(\rho+\gamma)}{\pi}\right]
$$

The optimal waiting time $t^{*}$ increases in $F$ : a higher entry cost justifies a longer waiting period on the part of the second entrant. Also, $t^{*}$ shrinks with $\pi$ since the opportunity cost of waiting increases as $\pi$ goes up. The positive externality parameter $\gamma$ has an interesting effect on $t^{*}$. On the one hand, a larger $\gamma$ makes waiting more attractive since the longer the firm waits the lower its cost of entry. On the other hand, a larger $\gamma$ leads to a lower cost of entry at any given point in time thereby making entry more attractive. Specifically, since $\frac{d t^{*}}{d \gamma}=\frac{1}{\gamma}\left[\frac{1}{\rho+\gamma}-t^{*}\right]$, it follows that $\frac{d t^{*}}{d \gamma}>0$ if and only if $t^{*}<\frac{1}{\rho+\gamma}$. For small $\gamma, t^{*}$ increases with $\gamma$. But if $\gamma$ is relatively large, then $t^{*}$ decreases with $\gamma$. 
Lemma 1: Suppose $\pi<(\rho+\gamma) F$. Then,

(i) $V_{1}\left(0, t^{*}\right)>V_{2}\left(0, t^{*}\right)$; and

(ii) there exists a $\hat{t} \in(0, \infty)$ such that firm i's best response function, denoted as $R\left(t_{j}\right)$, is given by

$$
R\left(t_{j}\right)=\left\{\begin{array}{ccc}
t_{j}+t^{*} & \text { if } & 0 \leq t_{j} \leq \hat{t} \\
0 & \text { if } & t_{j}>\widehat{t}
\end{array}\right\} .
$$

Proof: We know that

$$
V_{2} \equiv e^{-\rho t^{*}} \frac{\pi}{\rho}-F e^{-(\rho+\gamma) t^{*}} \text { and } V_{1} \equiv\left(1-e^{-\rho t^{*}}\right) \frac{m}{\rho}+e^{-\rho t^{*}} \frac{\pi}{\rho}-F
$$

where $t^{*}=\frac{1}{\gamma} \ln \left[\frac{(\rho+\gamma) F}{\pi}\right]$. We have

$$
V_{1}-V_{2}=\left(1-e^{-\rho t^{*}}\right) \frac{m}{\rho}-F\left(1-e^{-(\rho+\gamma) t^{*}}\right)=\left(1-x^{\rho / \gamma}\right) \frac{m}{\rho}-F\left(1-x^{\rho / \gamma+1}\right),
$$

where $x \equiv \frac{\pi}{F(\rho+\gamma)}<1$. Thus,

$$
\frac{d\left(V_{1}-V_{2}\right)}{d x}=-\frac{1}{\gamma} x^{\rho / r-1}[m-F(\rho+\gamma) x]=-\frac{1}{\gamma} x^{\rho / r-1}[m-\pi]<0 .
$$

This, together with the fact that $V_{1}=V_{2}$ when $x=1$, implies $V_{1}>V_{2}$ for all $0<x<1$. This proves part (i) of the lemma.

Clearly, if firm $i$ enters after firm $j$, it enters at time $t_{j}+t^{*}$, and if it enters before firm $j$, it enters at $t=0$. Which of the two options is better depends on the relative magnitudes of $V_{i}\left(0, t_{j}\right)$ and $V_{i}\left(t_{j}+t^{*}, t_{j}\right)$. At $t_{j}=0, V_{i}(0,0)<V_{i}\left(t^{*}, 0\right)$, by the definition of $t^{*}$. Since $V_{i}\left(0, t_{j}\right)$ increases with $t_{j}$, whereas $V_{i}\left(t_{j}+t^{*}, t_{j}\right)$ decreases with it and approaches zero as $t_{j}$ goes to $+\infty$, there exists $0<\widehat{t}<\infty$ such that $V_{i}(0, \widehat{t})=V_{i}\left(\widehat{t}+t^{*}, \widehat{t}\right)$. Thus, $V_{i}\left(0, t_{j}\right)<V_{i}\left(t_{j}+t^{*}, t_{j}\right)$ iff $t_{j}<\widehat{t}$.

While the first entrant earns monopoly profits till the second firms enters, the second entrant incurs a lower entry cost due to the positive externality generated by $\gamma$. Part (i) of Lemma 1 shows that the first entrant receives a higher payoff 
than the second. This result is later used to prove the existence of a sequential entry equilibrium. ${ }^{5}$

The properties of the reaction functions are also easy to understand. If firm $j$ enters at time 0 , then firm $i$ will choose to enter at $t^{*}$ in order to take advantage of the positive externality. If firm $j$ enters relatively early (i.e., $t_{j}$ is small), firm $i$ still prefers to delay entry by time $t^{*}$ and enters at time $t_{j}+t^{*}$, as long as $t_{j}$ is before a critical time $\hat{t}$. However, if $t_{j}>\hat{t}$, it is not worthwhile for firm $i$ to delay entry till $t_{j}+t^{*}$ and it prefers to enter at $t_{i}=0$ to capture the flow profit from time 0 . There exists a critical time $\hat{t}$ such that, given firm $j$ enters at time $\hat{t}$, firm $i$ is indifferent between entering at time 0 and waiting till time $\hat{t}+\hat{t}$, where $\hat{t}$ is defined by equation $V_{1}(0, \hat{t})=V_{1}\left(\widehat{t}+t^{*}, \hat{t}\right)$.

A sequential entry equilibrium must involve the first entrant entering at $t=0$ (from (3.2)). If firm 1 enters at $t=0$ then firm 2's optimal response is to enter at $t^{*}$. If firm 2 enters at $t^{*}$, firm 1 has two options: It either enters before $t^{*}$, in which case it enters at time 0 and receives a life-time payoff of $V_{1}\left(0, t^{*}\right)$. Alternatively, firm 1 can enter after $t^{*}$, in which case it enters at $2 t^{*}$ and earns a payoff of $V_{1}\left(2 t^{*}, t^{*}\right)$. Since $V_{1}\left(2 t^{*}, t^{*}\right)=e^{-\rho t^{*}} V_{2}\left(0, t^{*}\right)$ and from part (i) of lemma 1 we know that $V_{1}\left(0, t^{*}\right)>V_{2}\left(0, t^{*}\right)$. Thus, $V_{1}\left(0, t^{*}\right)>V_{1}\left(2 t^{*}, t^{*}\right){ }^{6}$ Therefore, $t_{1}=0$ is the best response to $t_{2}=t^{*}$ and consequently $\left(0, t^{*}\right)$ is a Nash equilibrium.

\footnotetext{
${ }^{5}$ Another interesting point is that the first entrant may benefit from an increase in the entry cost $(F)$. This is because a higher entry cost implies a longer waiting period on the part of the second entrant (see equation 3.4) and therefore a longer monopoly reign for the first entrant. To see this, note that

$$
\frac{d V_{1}\left(0, t^{*}\right)}{d F}=(m-\pi) e^{-\rho t^{*}} \frac{d t^{*}}{d F}-1
$$

so that an increase in $F$ has a direct negative effect (captured by -1 ) and an indirect positive effect (captured by the first term since $\frac{d t^{*}}{d F}>0$ ) on $V_{1}$. Using equation (3.4), we have:

$$
\frac{d V_{1}\left(0, t^{*}\right)}{d F}=\frac{(m-\pi)}{\gamma F^{1+\frac{\rho}{\gamma}}}\left[\frac{\pi}{\rho+\gamma}\right]^{\frac{\rho}{\gamma}}-1
$$

Thus, the indirect effect may dominate the direct negative effect when the monopoly flow profit $m$ is large (or when $F$ is small).

${ }^{6}$ This means that $t^{*}$ is always greater than the critical value $\widehat{t}$.
} 
Proposition 2: If $\rho F<\pi<(\rho+\gamma) F$, a unique equilibrium entry pattern exists in which one firm enters at time 0 and the other at time $t^{*}$.

The intuition behind Proposition 1 and Proposition 2 is simple: Firms enter simultaneously at date $t=0$ if the positive externality is not strong (so that $(\rho+\gamma) F<\pi))$. Otherwise, one firm delays its entry in order to enjoy the positive externality conferred by the entry of its rival.

\section{A symmetric Firms}

Suppose that the two firms differ in their marginal costs of production: firm 1 has a lower marginal cost of production than firm 2. Let $\pi_{1}$ and $\pi_{2}$ denote each firm's flow duopoly profits where $\pi_{1} \geq \pi_{2}$ and $m_{i}$ denote each firm's monopoly profits where $m_{1} \geq m_{2}$. Similar to Assumption 1, let $\pi_{2}>\rho F$. When firms are asymmetric, their incentives for entry do not coincide. In particular, the opportunity costs of delaying entry are higher for the low-cost firm: it forgoes $m_{1} \geq m_{2}$ for every instant it delays its entry given that firm 2 has not entered yet. One thus expects that it is more likely that the low-cost firm enters first.

\subsection{Pattern of Entry}

As before, when each firm's flow duopoly profits exceed the threshold $(\rho+\gamma) F$, the cost-lowering externalities are not large enough to induce either firm to delay entry, so to enter at time 0 is the dominant strategy for each firm. When $\pi_{1}>$ $(\rho+\gamma) F>\pi_{2}$, the cost-lowering externalities are not large enough for firm 1 to delay entry, but are sufficient for firm 2 to wait. In this case, to enter at $t_{1}=0$ is still the dominant strategy for firm 1 . Given this, firm 2 enters at its optimal time $t_{2}^{*}=\frac{1}{\gamma} \ln \left[\frac{F(\rho+\gamma)}{\pi_{2}}\right]$. The following result thus obtains.

Proposition 3: If $\pi_{2}>(\rho+\gamma) F$ simultaneous entry at $t=0$ is the unique Nash equilibrium. If $\pi_{2}<(\rho+\gamma) F<\pi_{1}$ then $\left(0, t_{2}^{*}\right)$ is the only Nash equilibrium. 
When $\pi_{1}<(\rho+\gamma) F$, both firms find delaying entry worthwhile. As a result, there may exist two different kinds of equilibria: one in which firm 1 enters first and the other in which it enters second. As in the previous section, given that firm $j$ enters at time $t_{j}^{*}$, for firm $i$ to be the first to enter in equilibrium, it must be that its payoff of entering at $t_{i}=0$ exceeds the payoff it would receive if it waits (after firm $j$ 's entry) for its optimal delay time and then enters at time $t_{j}^{*}+t_{i}^{*}$, where $t_{k}^{*}=\frac{1}{\gamma} \ln \left[\frac{F(\rho+\gamma)}{\pi_{\mathrm{k}}}\right], k=1,2$. Since firms are asymmetric, the conditions under which this restriction on payoffs is satisfied also differ. But, because delaying entry is relatively more costly for firm 1 , we can show that whenever the benefits of delaying entry are sufficient for firm 1 to enter later, they are also sufficient for delaying firm 2's entry.

Proposition 4: Let $\pi_{2} \leq \pi_{1}<(\rho+\gamma) F$. If $\left(t_{1}^{*}, 0\right)$ is a Nash equilibrium, then $\left(0, t_{2}^{*}\right)$ is a Nash equilibrium as well.

P roof. Let $\Delta_{1} \equiv V_{1}\left(0, t_{2}^{*}\right)-V_{1}\left(t_{1}^{*}+t_{2}^{*}, t_{2}^{*}\right)$ and $\Delta_{2} \equiv V_{2}\left(t_{1}^{*}, 0\right)-V_{2}\left(t_{1}^{*}, t_{1}^{*}+t_{2}^{*}\right)$. By definition,

$$
\Delta_{1}=\frac{1}{\rho}\left[1-e^{-\rho t_{2}^{*}}\right] m_{1}+\frac{e^{-\rho t_{2}^{*}}}{\rho}\left[1-e^{-\rho t_{1}^{*}}\right] \pi_{1}-F\left[1-e^{-\rho\left(t_{1}^{*}+t_{2}^{*}\right)-\gamma t_{1}^{*}}\right]
$$

and

$$
\Delta_{2}=\frac{1}{\rho}\left[1-e^{-\rho t_{1}^{*}}\right] m_{2}+\frac{e^{-\rho t_{1}^{*}}}{\rho}\left[1-e^{-\rho t_{2}^{*}}\right] \pi_{2}-F\left[1-e^{-\rho\left(t_{1}^{*}+t_{2}^{*}\right)-\gamma t_{2}^{*}}\right]
$$

For $\left(t_{1}^{*}, 0\right)$ to be a Nash equilibrium, there must be $\Delta_{2} \geq 0$ and for $\left(0, t_{2}^{*}\right)$ to be a Nash equilibrium, there must be $\Delta_{1} \geq 0$. Since $t_{1}^{*}<t_{2}^{*}$ and $\pi_{1} \geq \pi_{2}$ and $m_{1} \geq m_{2}$, the first two terms of $\Delta_{1}$ are greater than the corresponding terms of $\Delta_{2}$, and the third term of $\Delta_{1}$ is smaller than the corresponding term of $\Delta_{2}$. As a result, $\Delta_{1} \geq \Delta_{2}$. Consequently, if $\left(t_{1}^{*}, 0\right)$ is a Nash equilibrium, so is $\left(0, t_{2}^{*}\right)$.

Figure 1 below illustrates equilibrium outcomes for both the asymmetric case and the symmetric case. The figure is plotted in the $\left(\pi_{1}, \pi_{2}\right)$ space. The symmetric case is represented on the 45 degree line. In area $\mathrm{ABC}$, both $\left(0, t_{2}^{*}\right)$ and $\left(t_{1}^{*}, 0\right)$ 
could be Nash equilibria. To the right of BC and below CD, only $\left(0, t_{2}^{*}\right)$ is a Nash equilibrium and in the area above $\mathrm{CD}$ and below the 45 degree line, simultaneous entry is the Nash equilibrium. Since the region in which sequential entry occurs with the low-cost firm entering first contains the region in which the high-cost firm enters first, our results show that the low-cost firm is more likely to enter first in our model.

\section{— Insert figure 1 here -}

\subsection{Industry Profits under Sequential Entry}

The question we address here is the following: among the two Nash equilibria $\left(0, t_{2}^{*}\right)$ and $\left(t_{1}^{*}, 0\right)$, which one leads to higher industry profits?

Let $V\left(0, t_{2}^{*}\right)$ and $V\left(t_{1}^{*}, 0\right)$ denote the industry profits in these two equilibria, respectively. We have

$$
V\left(0, t_{2}^{*}\right)=\int_{0}^{t_{2}^{*}} e^{-\rho t} m_{1} d t+\int_{t_{2}^{*}}^{+\infty} e^{-\rho t}\left[\pi_{1}+\pi_{2}\right] d t-F\left[1+e^{-(\rho+\gamma) t_{2}^{*}}\right]
$$

and

$$
V\left(t_{1}^{*}, 0\right)=\int_{0}^{t_{1}^{*}} e^{-\rho t} m_{2} d t+\int_{t_{1}^{*}}^{+\infty} e^{-\rho t}\left[\pi_{1}+\pi_{2}\right] d t-F\left[1+e^{-(\rho+\gamma) t_{1}^{*}}\right]
$$

Since $t_{2}^{*} \geq t_{1}^{*}, m_{1} \geq m_{2}$ and $m_{1}>\left[\pi_{1}+\pi_{2}\right]$, it must be that $V\left(0, t_{2}^{*}\right)>V\left(t_{1}^{*}, 0\right)$. That is, total industry profits are higher in the sequential equilibrium in which the low-cost firm enters first. This is so because of three reasons. First, since $m_{1} \geq m_{2}$, the low-cost monopolist makes higher profits till the second firm enters. Second, since $t_{2}^{*} \geq t_{1}^{*}$ monopoly position is maintained longer in equilibrium $\left(0, t_{2}^{*}\right)$ than in $\left(t_{1}^{*}, 0\right)$. Finally, entry cost that is paid in equilibrium $\left(0, t_{2}^{*}\right)$ is lower because the high-cost firm stays out of the market for a longer time and pays a lower entry cost. Hence, if the two firms could make side payments, we would expect the low-cost firm to enter first. 


\section{Welfare A nalysis}

Regarding the welfare properties of different entry patterns that exist in this model, one can ask two types of questions. First, do firms enter too early or too late from society's standpoint? Second, when firms differ in cost, is it necessarily the case that social welfare is higher when the low-cost firm enters first?.

First, regarding entry times, it is clear that in simultaneous entry equilibrium, firms never enter too early in this model. This is because firms internalize the benefits of delaying entry and if they prefer not to delay entry, neither does society.

In a given sequential entry equilibrium, say $\left(0, t_{2}^{*}\right)$, is $t_{2}^{*}$ is the socially optimal waiting period? Given that firm 1 enters at $t=0$, the socially optimal entry time of firm 2 is given by:

$$
t_{s} \equiv \underset{t}{\operatorname{ArgMax}} W(t)=V_{1}(0, t)+V_{2}(0, t)+\int_{0}^{t} e^{-\rho t} C_{1} d t+\int_{t}^{+\infty} e^{-\rho t} C_{2} d t
$$

where $C_{1}$ denotes the consumer surplus when only firm 1 is in the market and $C_{2}$ is the consumer surplus under duopoly. We can write $W(t)$ as $W(t)=$ $e^{-\rho t}\left[\frac{\Lambda}{\rho}-F e^{-\gamma t}\right]+\frac{m_{1}+C_{1}}{\rho}-F$, where $\Lambda \equiv \pi_{1}+\pi_{2}+C_{2}-m_{1}-C_{1}$ is the amount by which total surplus under duopoly exceeds the total surplus under monopoly. Taking the first order condition and solving for $t_{s}$ we get $t_{s}=\frac{1}{\gamma} \ln \left[\frac{F(\rho+\gamma)}{\Lambda}\right]$. Comparing $t_{s}$ with $t_{2}^{*}$ we find that $t_{2}^{*} \geq t_{s}$ if and only if $\Lambda \geq \pi_{2}$.

P roposition 5: In a sequential entry equilibrium, the second firm enters too late if and only if $\Lambda \geq \pi_{2}$.

There are two opposing forces that affect the private entry time vis-a-vis the socially optimal entry time. On the one hand, when delaying its entry the second entrant does not take into account the additional consumer surplus that is generated as a result of its entry. On the other hand, the second entrant also fails to take into account the fact that its entry erodes the first entrant's monopoly status. In general, either of those two effects can dominate. For linear demand $P=A-Q$ and symmetric (Cournot) firms with zero production cost, it follows 
that $m_{1}=m_{2}=A^{2} / 4, C_{1}=A^{2} / 8, \pi_{1}=\pi_{2}=A^{2} / 9$, and $C_{2}=2 A^{2} / 9$. As a result, $\Lambda=5 A^{2} / 72<\pi$. Thus, the second firm enters too early in this case.

If the firms are asymmetric, either of them may enter first in equilibrium. When the low-cost firm enters first (in the equilibrium $\left(0, t_{2}^{*}\right)$ ), the flow monopoly profit and consumer surplus prior to the second firm's entry are higher than in the equilibrium $\left(t_{1}^{*}, 0\right)$ where the high-cost firm enters first. Also, since $t_{2}^{*}>t_{1}^{*}$, entry cost is lower in the first equilibrium as the high-cost firm waits longer before its entry in order to take the advantage of positive externality generated by the lowcost firm's entry. However, for the same reason, monopoly position is maintained for a longer period of time in the first equilibrium. In general, it is hard to rank these two equilibria in terms of social welfare. ${ }^{7}$

\section{Conclusion}

In this paper we endogenize the pattern of entry where initial entry generates positive externalities for other potential entrants. We derive conditions which lead to simultaneous or sequential entry and demonstrate the robustness of the results when the firms differ in cost. Our model provides several new insights. For symmetric firms, an increase in entry cost may actually benefit the first entrant since it delays entry by its rival firm (see footnote 5). Furthermore, the optimal entry time of the second firm is not monotonic in the magnitude of the externality from the first firm's entry. Under the asymmetric case we show that while there is no guarantee that the low-cost firm enters the market first (socially desirable), it is nevertheless the case that the low-cost firm is more likely to enter before the high-cost firm. We also find that the optimal entry time of the second entrant does not necessarily exceed the socially optimal entry time, as might be expected in the face of a positive externality.

An important assumption made in our model is that firms are able to com-

\footnotetext{
${ }^{7}$ Simulations results for linear demand indicate that for various combinations of parameter values (the cost differential between the two firms, the magnitude of $\gamma$, the fixed entry cost $F$, and the discount factor $\rho$ ), social welfare is higher when the low-cost firm enters first.
} 
mit to their entry times at $t=0$. This commitment assumption simplifies the derivations of the equilibrium entry patterns and enables us to focus on the basic trade-off between entering first and earning monopoly profit versus delaying entry to lower the cost of entry. In Lin and Saggi (2001), we extend our model to a dynamic game where firms are unable to commit to entry times. ${ }^{8}$ We show that the results obtained in the present model are not an artifact of the commitment assumption: the simultaneous entry and sequential entry equilibria obtained in this model are also subgame perfect equilibria in the dynamic model. The dynamic model does offer one additional insight though. In a dynamic setting, there exists a coordination problem between firms: the incentive to enter first coupled with the incentive to avoid simultaneous entry can result in a Pareto-inferior equilibrium in which each firm chooses not to enter with a positive probability in each time period $t$, conditional on no entry by that time. If firms could coordinate their entry decisions, they would choose the entry pattern described by the sequential entry equilibrium of our model.

\section{R eferences}

[1] Aitken, Brian, Gordon H. Hanson, and Ann E. Harrison (1997): "Spillovers, Foreign Investment, and Export Behavior." J ournal of International Economics 43: 103-132.

[2] Appelbaum, Elie and Shlomo Weber (1994): "Equilibrium Entry Patterns under Uncertainty." E uropean E conomic Review 38: 45-58.

[3] Business Week, March 4, 1996: 56-64.

[4] Lin, P. and K. Saggi (2001): "Notes on the Timing of Entry under Externalities." Working Paper, Lingnan University.

\footnotetext{
${ }^{8}$ The model we consider in that paper is a dynamic discrete time model in which firms choose whether or not to enter the market in every time period. Because of technical complexity, we did not study a differential game in continuous time.
} 
[5] Maggi, G. (1996): "Endogenous Leadership in a New Market." Rand J ournal of Economics, 27: 641-59.

[6] Reinganum, J. (1981a): "On the Diffusion of New Technology: A GameTheoretic Approach." Review of E conomic Studies, 48: 395-406.

[7] Reinganum, J. (1981b): "Market Structure and the Diffusion of New Technology." Bell J ournal of Economics 12: 618-624.

[8] Rob, Rafael. (1990): "Learning and Capacity Expansion under Demand Uncertainty." Review of Economic Studies 58: 655-675.

[9] Rob, Rafael. (1992): "Sales, Uncertainty and the Determinants of Investment." mimeo, CARESS working paper 92-03, University of Pennsylvania. 